\title{
Influência dos bicos artificiais na amamentação em lactentes atendidos em um banco de leite humano
}

\author{
Influence of artificial nozzles on breastfeeding in infants treated at a human milk bank \\ Influencia de las boquillas artificiales en la lactancia materna en bebés tratados en un \\ banco de leche humana
}

Tahisa Ferreira da Silva ${ }^{1 *}$, Sandra Hipólito Cavalcanti ${ }^{1}$, Bruna Rafaela Ferreira da Silva Lima², Talita Munique de Melo Rodrigues ${ }^{1}$, Luana Marques Avelino Cavalcanti ${ }^{4}$, Carla Tacilia Bezerra ${ }^{3}$, Isabella Eduarda de Melo Oliveira ${ }^{5}$, Débora Beatriz Nascimento Almeida Gama ${ }^{6}$, Karla Pires Moura Barbosa $^{3}$, Letícia Moura de Vasconcelos ${ }^{1}$.

\section{RESUMO}

Objetivo: Identificar a influência dos bicos artificiais na amamentação das crianças atendidas em um Banco de Leite Humano (BLH) de um Hospital Amigo da Criança em um município de Pernambuco. Métodos: Estudo retrospectivo de corte transversal com abordagem quantitativa com uma amostra de 413 atendimentos. No tratamento estatístico foi utilizada a análise uni e bivariada para obtenção da distribuição dos valores de frequência absoluta e relativa e o teste Qui-quadrado na associação das variáveis a fim de determinar o $p$ valor. Resultados: Quase todas as puérperas realizaram acompanhamento pré-natal $(99,5 \%)$, porém apenas $33,4 \%$ receberam orientações sobre amamentação durante as consultas. As crianças do estudo tinham em média 13,61 dias de vida e 38,7\% já haviam utilizado bicos artificiais, com maior prevalência das chupetas, verificando que esses bicos influenciaram na pega incorreta e problemas com a mama. Conclusão: $O$ baixo percentual de orientações sobre amamentação no pré-natal pode ter favorecido ao aumento do percentual de utilização de bicos artificiais, desta forma é de suma importância que haja enfermeiros qualificados para atuar junto à gestante/puérpera no processo de educação em saúde, afim de reduzir os efeitos deletérios desta prática.

Palavras-chave: Aleitamento materno, Chupeta, Mamadeiras.

\section{ABSTRACT}

Objective: To identify the influence of artificial teats on the breastfeeding of children attended at a Human Milk Bank (HMB) of a Baby Friendly Hospital in a municipality in Pernambuco. Methods: Retrospective crosssectional study with a quantitative approach with a sample of 413 consultations. In the statistical treatment, univariate and bivariate analysis were used to obtain the distribution of absolute and relative frequency values and the chi-square test in the association of variables to determine the $p$-value. Results: Almost all mothers had antenatal care $(99.5 \%)$, but only $33.4 \%$ received guidance on breastfeeding during consultations. The children in the study had an average of 13.61 days of life and $38.7 \%$ had already used artificial nipples, with a higher prevalence of pacifiers, verifying that these nipples influenced the incorrect grip and problems with the breast. Conclusion: The lowpercentage of guidelines on breastfeeding in prenatal care may have favored the increase in the percentage of use of artificial nipples, so it is extremely important that there are qualified nurses to work with the pregnant / postpartum woman in the health education process in order to reduce the harmful effects of this practice.

Keywords: Breast feeding, Pacifiers, Nursing bottles.

1 Instituto de Medicina Integral Professor Fernando Figueira (IMIP), Recife - PE.

${ }^{*}$ E-mail: tahisa.silva@gmail.com

${ }^{2}$ Faculdade de Ciências Médicas de Pernambuco - FCM/UPE, Recife - PE.

${ }^{3}$ Universidade Federal de Pernambuco (UFPE), Recife - PE.

${ }^{4}$ Secretaria de Saúde de Jaboatão dos Guararapes, Jaboatão dos Guararapes - PE.

${ }^{5}$ Secretaria de Saúde do Recife, Recife - PE.

${ }^{6}$ Centro Universitário Internacional (UNINTER), Recife - PE. 


\section{RESUMEN}

Objetivo: Identificar la influencia de las tetinas artificiales en la lactancia materna de niños atendidos en un Banco de Leche Humana (HMB) de un Hospital Amigo del Niño en un municipio de Pernambuco. Métodos: Estudio transversal retrospectivo con enfoque cuantitativo con una muestra de 413 consultas. En el tratamiento estadístico, se utilizaron análisis uni y bivariados para obtener la distribución de los valores de frecuencia absoluta y relativa y la prueba de Chi-cuadrado en la asociación de variables para determinar el valor p. Resultados: Casi todas las mujeres puerperales recibieron atención prenatal (99.5\%), pero solo el $33.4 \%$ recibió orientación sobre la lactancia durante las consultas. Los niños en el estudio tenían un promedio de 13.61 días de vida y el $38.7 \%$ ya habían usado tetinas artificiales, con una mayor prevalencia de chupetes, verificando que estas tetinas influyeron en el agarre incorrecto y los problemas con el seno. Conclusión: El bajo porcentaje de pautas sobre lactancia materna en la atención prenatal puede haber favorecido el aumento en el porcentaje de uso de pezones artificiales, por lo que es extremadamente importante que haya enfermeras calificadas para trabajar con la mujer embarazada / posparto en el proceso de educación sanitaria, para para reducir los efectos nocivos de esta práctica.

Palabras clave: Lactancia materna, Chupetes, Biberones.

\section{INTRODUÇÃO}

O aleitamento materno exclusivo (AME) é recomendado pela Organização Mundial de Saúde (OMS), até os seis meses de idade da criança, após esse período deve ser complementado com alimentos saudáveis e mantido até os 2 anos ou mais. É conhecido cientificamente que o leite materno além de proteger a criança contra diarreias, pneumonias, infecções e alergias, propicia melhor desenvolvimento do sistema nervoso, forte vínculo com a mãe e diminui os riscos de desenvolver diabetes, obesidade, hipertensão arterial e vários tipos de câncer na vida adulta (MINISTÉRIO DA SAÚDE, 2015; TOMA TS e REA MF, 2008).

Além dos efeitos positivos para a criança, também existem benefícios maternos que incluem a proteção contra diversas doenças, por exemplo: o câncer de mama, diabetes tipo 2, hipertensão arterial, doenças metabólicas, entre outras. O AME além disso, propicia um efeito anticoncepcional conhecido como amenorreia lactacional, devido ao estímulo de sucção e aumento da prolactina, e também auxilia na involução uterina no período do puerpério e recuperação do peso pré-gestacional (MINISTÉRIO DA SAÚDE, 2015; REA $\mathrm{MF}, 2004)$.

Em outubro de 2008, foi realizado a II Pesquisa de Prevalência do Aleitamento Materno (AM) nas Capitais Brasileiras e Distrito Federal, que traz como resultado, a mediana do AME igual a $41 \%$ e do AM de 11,2 meses, nas crianças menores de 12 meses, no país. Esses resultados são classificados segundo a OMS como razoável e ruim, respectivamente (MINISTÉRIO DA SAÚDE, 2009).

Para que seja alcançado um parâmetro muito bom, a prevalência do AME deveria ser de 90 a 100\% e a duração mediana da AM entre 23 e 24 meses, o que ressalta a importância de investimentos em políticas públicas, a fim de melhorar os índices do país e consequentemente a saúde da população infantil e diminuição dos índices de internamento (MINISTÉRIO DA SAÚDE, 2015).

Dentre os vários fatores que podem influenciar no insucesso da amamentação, o mais prevalente é o uso de chupeta e mamadeira, também denominado de "bicos artificiais". O estudo de Batista CLC, et al. (2018); analisou cinco aspectos da mamada: posição mãe/bebê, resposta da dupla, adequação da sucção, anatomia das mamas e afetividade, e obteve como resultado que os bebês que utilizavam bicos artificiais apresentaram escores ruins elevados em todos os aspectos, com destaque para a adequação da sucção.

Inúmeras são as implicações do uso dos bicos artificiais, a chupeta, por exemplo, pode prejudicar a maturação do sistema estomatognático alterando a postura e tonicidade dos músculos da face, e a sucção não nutritiva pode proporcionar uma saciedade neural, o que implica em uma menor demanda na amamentação, resultando em menor estimulação mamária e consequentemente diminuição da produção de leite e ganho ponderal por vezes inadequado (SOCIEDADE BRASILEIRA DE PEDIATRIA, 2017). 
Devido a alterações na musculatura orofacial pode estar presente a dificuldade em fechar os lábios, hipotonia lingual e a respiração bucal, a arcada dentária forma-se como um arco e a fala também pode ser alterada, além da repercussão social, pois no momento em que a criança é inserida na escola utilizando estes artefatos há uma menor interação e desenvolvimento social (ARAÚJO CMT, et al., 2007)

As alterações na musculatura da face e a cavidade oral se dão em consequência da adaptação à sucção dos bicos artificiais, este fator pode causar um fenômeno conhecido como confusão de bicos, que é caracterizado pela dificuldade da criança em assumir a amamentação de forma efetiva após a exposição à mamadeiras e chupetas. A criança passa a ter dificuldade em assumir uma conformação oral adequada, ou seja, abertura da boca, posicionamento da língua, padrão de sucção e pega eficaz (BATISTA CLC, et al., 2017).

A mastigação e respiração também são afetadas, a mastigação tende a ser vertical, essa alteração afeta as articulações e outras estruturas envolvidas o que resulta numa deglutição atípica, já na respiração o padrão respiratório se altera para oral ou misto. Não esquecendo do sistema imunológico, chupetas e mamadeiras são consideradas reservatórios de infecção, o que afeta diretamente o sistema imunológico da criança, aumentando o risco de diarreias e a probabilidade de internações (SOCIEDADE BRASILEIRA DE PEDIATRIA, 2017).

Crianças que utilizam chupeta demandam menos atenção dos pais, aparentemente encontram-se mais "calmas", levando a uma menor estimulação e por sua vez podendo alterar no desenvolvimento. Já existem estudos afirmando que pessoas que utilizavam chupeta na infância apresentaram um desempenho menor comparado àquelas que não possuíam esse hábito (SOCIEDADE BRASILEIRA DE PEDIATRIA, 2017).

Reconhecendo a importância e os vários fatores que influenciam no insucesso do AME, a OMS desaconselha, nos "Dez passos para o Sucesso do Aleitamento Materno", a utilização de bicos artificiais. Apesar de contraindicados, os índices de uso dos bicos artificiais ainda permanecem elevados, sendo de $58,4 \%$ para mamadeiras e 42,6\% para o uso de chupetas (MINISTÉRIO DA SAÚDE, 2015; MINISTÉRIO DA SAÚDE, 2009).

Estudos atuais mostram que os fatores determinantes para a utilização dos bicos artificiais são, principalmente, crianças de baixo peso ao nascer (BPN), não amamentadas na maternidade, choro excessivo e introdução de outros líquidos. Já entre os determinantes maternos estão as mulheres primíparas, adolescentes, inseguras na amamentação, retorno ao trabalho e convivência com a avó materna (BUCCINI GS, et al., 2014).

É de suma importância uma assistência de qualidade desde o pré-natal até o nascimento da criança, incentivando e aconselhando a importância do aleitamento materno. Os hospitais que cumprem os "Dez passos para o Sucesso do Aleitamento Materno", recebem um selo de qualidade do Ministério da Saúde (MS) intitulado: Iniciativa Hospital Amigo da Criança (IHAC), para tal se faz necessário, também, cumprir a Norma Brasileira de Comercialização de Alimentos para Lactentes e Crianças na Primeira Infância, Bicos, Chupetas e Mamadeira (NBCAL) e prestar uma assistência humanizada a mulher (PORTAL MINISTÉRIO DA SAÚDE, 2019).

Diante dos riscos relacionados aos bicos artificiais, descritos na literatura, com elevado potencial ao desmame da amamentação, este estudo objetiva identificar a influência dos bicos artificiais na amamentação das crianças atendidas em um Banco de Leite Humano (BLH) de um Hospital Amigo da Criança localizado em um município de Pernambuco.

\section{MÉTODOS}

Trata-se de um estudo retrospectivo de corte transversal com abordagem quantitativa, realizado no BLH no mês de julho/2019, após a aprovação do Comitê de Ética e Pesquisa da instituição, sob o parecer CAAE 13671319.7.0000.5201. Os dados foram extraídos das fichas de atendimento das nutrizes atendidas no BLH, nos meses de novembro e dezembro de 2018 , através de um formulário estruturado. 
O número total de atendimentos realizados nos meses supracitados foi igual a 591 , porém só foram incluídos no estudo as fichas de atendimento que possuíam a maioria dos dados sobre a avaliação da amamentação devidamente registrados e os atendimentos realizados na presença do recém-nascido (RN) /lactente, isto resultou numa amostra final de 413 atendimentos, porém estas fichas de atendimento ainda possuíam alguns dados incompletos e estes aparecem nas tabelas como a categoria "não respondido".

O banco de dados foi criado no programa EPIINFO, versão 3.5.2, e exportado para o software IBM® SPSSStatistical Package for the Social Sciences, versão 18. No tratamento estatístico foi utilizada a análise univariada e bivariada para obtenção da distribuição dos valores de frequência absoluta e relativa, foi utilizado também, o teste Qui-quadrado na associação das variáveis a fim de apresentar o $p$-valor, o qual deve ser $<0,05$, para determinar significância entre as variáveis. Os dados obtidos foram apresentados em forma de tabelas e a análise teve como base o referencial bibliográfico de relevância e pertinente ao tema.

\section{RESULTADOS}

Destacamos a importância de apresentar as características socioeconômicas maternas, obstétricas, características relacionadas à amamentação e ao uso de bicos artificiais, a fim de obter-se o impacto do uso dos bicos artificiais na amamentação e saúde infantil como é, também, descrito na literatura. Em relação às características maternas, foi possível observar que $86,9 \%$ tinham idade superior a 20 anos, não trabalhavam formalmente 57,4\% e 79,7\% residiam na Região Metropolitana de Recife (RMR) (Tabela 1).

No que diz respeito a realização do pré-natal, 99,5\% tiveram esse acompanhamento, o qual foi realizado, principalmente, em estabelecimentos da rede pública de saúde 44,1\% (Unidades Básicas de Saúde, policlínicas e hospitais) com o número de consultas superior a seis, 65,1\%, nas quais apenas 33,4\% receberam orientações sobre a amamentação durante as consultas de pré-natal (Tabela 1). 
Tabela 1 - Características socioeconômicas e obstétricas das puérperas atendidas no Banco de Leite Humano de um Hospital Amigo da Criança nos meses de novembro e dezembro de 2018.

\begin{tabular}{|c|c|c|}
\hline Variável & $\mathbf{N}$ & $\%$ \\
\hline \multicolumn{3}{|l|}{ Idade materna } \\
\hline Menor ou igual a 20 anos & 53 & 12,8 \\
\hline Maior que 20 anos & 359 & 86,9 \\
\hline Não respondido & 1 & 0,2 \\
\hline \multicolumn{3}{|l|}{ Trabalha } \\
\hline Sim & 174 & 42,1 \\
\hline Não & 237 & 57,4 \\
\hline Não respondido & 2 & 0,4 \\
\hline \multicolumn{3}{|l|}{ Local da residência } \\
\hline Região metropolitana de Recife (RMR) & 329 & 79,7 \\
\hline Interior & 84 & 20,3 \\
\hline \multicolumn{3}{|l|}{ Realizou acompanhamento pré-natal? } \\
\hline Sim & 411 & 99,5 \\
\hline Não & 1 & 0,2 \\
\hline Não respondido & 1 & 0,2 \\
\hline \multicolumn{3}{|l|}{ Local do pré-natal } \\
\hline IMIP & 110 & 26,6 \\
\hline Rede pública (USF, policlínicas...) & 182 & 44,1 \\
\hline IMIP + Rede pública & 28 & 6,8 \\
\hline Rede privada & 74 & 17,9 \\
\hline Rede pública + rede privada & 11 & 2,6 \\
\hline Não respondido & 7 & 1,7 \\
\hline Não se aplica & 1 & 0,2 \\
\hline \multicolumn{3}{|l|}{ Número de consultas } \\
\hline Menor ou igual a 6 & 118 & 28,6 \\
\hline Maior que 6 & 269 & 65,1 \\
\hline Não respondido & 25 & 6,1 \\
\hline Não se aplica & 1 & 0,2 \\
\hline \multicolumn{3}{|c|}{ Recebeu orientação sobre amamentação no pré-natal? } \\
\hline Sim & 138 & 33,4 \\
\hline Não & 188 & 45,5 \\
\hline Não respondido & 86 & 20,8 \\
\hline Não se aplica & 1 & 0,2 \\
\hline Total & 413 & - \\
\hline
\end{tabular}

Fonte: Silva TF, et al., 2019.

A maioria dos partos ocorreu com idade gestacional superior a 37 semanas $(77,7 \%)$ e o peso foi maior que 2500 gramas em $86,2 \%$, o que caracteriza RN a termo peso adequado ao nascer, respectivamente (MINISTÉRIO DA SAÚDE, 2017). O contato pele a pele (CPP) na sala de parto e a amamentação na primeira hora de vida (APH) são medidas consideradas importantes na promoção do aleitamento materno exclusivo, neste estudo foi observada uma frequência de $62,5 \%$ e $30,5 \%$ respectivamente. Os principais motivos da não adoção destas medidas, segundo resposta das mães na ficha de atendimento, foi o desconforto respiratório dos seus filhos no momento do nascimento, sendo $3,4 \%$ no CPP e $9,9 \%$ na APH, e o parto cesariana responsável por $1,7 \%$ no $\mathrm{CPP}$ e $\mathrm{APH}$.

Vale ressaltar que houve um grande número de "não respondidos" nestas variáveis. As crianças estudadas tinham idade entre 2 e 150 dias, com média de 13,61 dias e desvio padrão de 16,16, ou seja, eram menores de seis meses de idade e a taxa de aleitamento materno exclusivo foi de $24,5 \%$, os bicos artificiais tinham sido introduzidos em 160 (38,7\%) das mesmas (Tabela 2). 
Tabela 2 - Frequência de uso e tipos dos bicos artificiais utilizados pelas crianças atendidas no Banco de Leite Humano de um Hospital Amigo da Criança nos meses de novembro e dezembro de 2018.

\begin{tabular}{ccc}
\hline Tipos de bicos artificiais utilizados & N & $\%$ \\
\hline Chupeta & 77 & 18,6 \\
Mamadeira & 42 & 10,2 \\
Chupeta e mamadeira & 41 & 9,9 \\
\hline Total & 160 & -
\end{tabular}

Fonte: Silva TF, et al., 2019.

Alguns fatores relacionados à amamentação eram analisados no momento da consulta, por exemplo, pega e posição, fluxo e trauma mamário. A pega e posição apresentaram-se corretas em $51,3 \%$ e $55,2 \%$, respectivamente, o fluxo estava ótimo em $40,2 \%$ e o trauma mamilar foi relatado por cerca de $37,5 \%$ das genitoras (Tabela 3).

Tabela 3 - Avaliação dos fatores relacionados à amamentação associados ao binômio mãe-bebê.

\begin{tabular}{ccc}
\hline Variável & N & $\%$ \\
\hline Pega & 212 & 51,3 \\
Correta & 191 & 46,2 \\
Incorreta & 10 & 2,4 \\
Não respondido & & 55,2 \\
\hline Posição & 228 & 42,4 \\
\hline Correta & 175 & 2,4 \\
Incorreta & 10 & 40,2 \\
Não respondido & & 53,5 \\
\hline Fluxo mamário & 166 & 3,1 \\
\hline Ótimo & 221 & 1,9 \\
Bom & 13 & 1,2 \\
\hline Regular & 8 & \\
Insuficiente & 5 & 37,5 \\
Não respondido & & 62,0 \\
\hline mama & & 0,5 \\
\hline Sim & 155 & - \\
\hline Não & 256 &
\end{tabular}

Fonte: Silva TF, et al., 2019.

Os problemas com a mama encontrados foram: fissura mamilar (28,8\%), mamilos róseos $(10,7 \%)$, mamas túrgidas $(5,2 \%)$, áreas endurecidas na mama $(0,6 \%)$, excedente lácteo $(0,2 \%)$, hiperemia $(0,2 \%)$, dor ao amamentar $(0,2 \%)$, em relação ao bebê $0,4 \%$ apresentou monilíase oral. Foi realizado associação das variáveis a fim de verificar se o uso dos bicos artificiais tem influência na pega e posição no momento da amamentação, para que haja significância o $p$-valor deve ser $<0,05$.

Podemos observar que houve influência dos bicos artificiais na pega, ou seja, as crianças que utilizavam estes artefatos apresentaram predomínio de pega incorreta, o que foi confirmado pelo $p$-valor 0,012 . Já no caso da posição incorreta, predominantemente, nas crianças que utilizaram mamadeiras isoladamente ou em conjunto com as chupetas, o p-valor não demonstrou significância 0,052 (Tabela 4). 
Tabela 4 - Associação do uso de bicos artificiais com a pega e posição no momento da amamentação.

\begin{tabular}{|c|c|c|c|c|c|c|}
\hline \multirow[b]{2}{*}{ Variável } & \multicolumn{2}{|c|}{ Pega } & \multirow[b]{2}{*}{$\mathrm{p}$-valor } & \multicolumn{2}{|c|}{ Posição } & \multirow[b]{2}{*}{ p-valor } \\
\hline & $\begin{array}{c}\text { Correta } \\
\text { N (\%) }\end{array}$ & $\begin{array}{c}\text { Incorreta } \\
\mathbf{N}(\%)\end{array}$ & & $\begin{array}{c}\text { Correta } \\
\text { N (\%) }\end{array}$ & $\begin{array}{c}\text { Incorreta } \\
\mathbf{N}(\%)\end{array}$ & \\
\hline Chupeta & $37(48,1 \%)$ & $39(50,6 \%)$ & & $39(50,6 \%)$ & $36(46,8 \%)$ & \\
\hline Mamadeira & $16(38,1 \%)$ & $26(61,9 \%)$ & 0,012 & $19(45,2 \%)$ & $23(54,8 \%)$ & 0,052 \\
\hline Chupeta e mamadeira & $14(34,1 \%)$ & $25(61,0 \%)$ & & $17(41,5 \%)$ & $22(53,7 \%)$ & \\
\hline
\end{tabular}

Fonte: Silva TF, et al., 2019.

Houve associação, também, entre o uso dos bicos artificiais e a presença de trauma mamilar, pode-se observar que houve significância na presença de trauma mamilar quando as crianças utilizavam em conjunto mamadeiras e chupetas, a associação é corroborada pelo $p$-valor igual a 0,002 (Tabela 5).

Tabela 5 - Associação do uso de bicos artificiais com a presença de trauma mamário.

\begin{tabular}{cccc}
\hline & \multicolumn{2}{c}{ Trauma mamário } & \multirow{2}{*}{ p-valor } \\
\cline { 2 - 3 } Variável & $\mathbf{S i m}$ & $\mathbf{N a ̃ o}$ & \\
& $\mathbf{N}(\%)$ & $39(50,6 \%)$ & \\
\hline Chupeta & $38(49,4 \%)$ & $27(64,3 \%)$ & 0,002 \\
Mamadeira & $15(35,7 \%)$ & $19(46,3 \%)$ & \\
Chupeta e mamadeira & $20(48,8 \%)$ & 85 & - \\
\hline Total & 73 &
\end{tabular}

Fonte: Silva TF, et al., 2019.

\section{DISCUSSÃO}

De acordo com a Portaria ํㅡ 569, de $1^{\circ}$ de junho de 2000, que institui o Programa de Humanização no Pré-natal e Nascimento, no âmbito do Sistema Único de Saúde, existem alguns princípios e condições que visam garantir um acompanhamento pré-natal adequado, entre eles está a realização de atividades educativas (MINISTÉRIO DA SAÚDE, 2000). As práticas educativas proporcionam à gestante empoderamento acerca das diversas mudanças que acontecem em seu corpo durante a gestação e cuidados futuros com o RN, inclusive a amamentação. Dias EG, et al. (2015), demonstra a percepção das gestantes acerca da educação em saúde realizada no pré-natal, as quais estão satisfeitas com este ambiente de aprendizagem e ressaltaram esses momentos como uma oportunidade de fortalecer os conhecimentos, autocuidado e segurança em relação aos cuidados com o bebê.

É indiscutível a importância da educação em saúde, mas para que esta aconteça de forma efetiva faz-se necessário profissionais qualificados para atuarem com segurança e empoderamento no compartilhamento de informações e até mesmo desmistificação de crenças populares sobre a amamentação. Porém este cenário não está presente em unanimidade, Dominguez CC, et al. (2017), entrevistou enfermeiros atuantes em Unidades Básicas de Saúde, evidenciando que estes profissionais reconhecem que existe uma grande falha nas consultas de pré-natal em relação ao apoio e incentivo à amamentação, isto é relatado como consequência da insegurança e falta de capacitação profissional.

Marinho MS, et al. (2015), ratifica esta falta de capacitação em seu estudo, o mesmo afirma que as ações educativas, no âmbito do aleitamento materno, devem ser efetuadas no pré-natal e são vistas como instrumentos que incentivam e favorecem a adesão das genitoras à amamentação. No entanto, essas ações não ocorrem e apresentam como causa a ausência de atualização, por parte dos profissionais que prestam assistência durante o período gestacional, acarretando em atitudes maternas inapropriadas como, por exemplo, o desmame precoce. Foi observado neste estudo que apenas $33,4 \%$ receberam orientações sobre a amamentação durante as consultas de pré-natal, um ponto negativo que proporciona fragilidade no conhecimento e abertura à realização de práticas impróprias, isto pode ser um reflexo da falta de capacitação e conhecimento por parte dos profissionais atuantes junto à gestante. 
O CPP é uma importante medida que promove o aleitamento materno e está presente entre os "Dez passos para o sucesso do aleitamento materno", especificamente, é o quarto passo, que orienta a colocar os bebês em contato pele a pele na primeira hora após o parto e auxiliar a mãe a identificar os sinais se o bebê quer ser amamentado oferecendo o apoio necessário, além de promover a amamentação esta medida ajuda, também, na adaptação à vida extrauterina (ABDALA LG e DA CUNHA MLC, 2018; MOREIRA MEL, et al., 2014).

Um estudo nacional realizado pela Fiocruz, Nascer no Brasil, analisou as boas práticas realizadas em recém-nascidos saudáveis em todas as regiões do Brasil, na região nordeste, a prevalência do CPP foi de $28,8 \%$ e a amamentação na primeira hora de vida $41,3 \%$, o que mostra que há muito a melhorar neste âmbito e ressalta a importância da continuidade das ações de educação em saúde (MOREIRA MEL, et al., 2018).

O CPP e a amamentação na primeira hora de vida constatados neste estudo foi respectivamente, $62,5 \%$ e $30,5 \%$, esta taxa de aleitamento é considerada pelo MS como razoável, assim como a prevalência de AME em menores de seis meses (MINISTÉRIO DA SAÚDE, 2019).

Semelhante a presente pesquisa, segundo parâmetros da OMS, Silva JLP, et al. (2018), traz em seu estudo como a amamentação na primeira hora de vida não ocorre nos padrões adequados recomendados por essa organização em saúde, assim como o CPP.

Foi evidenciado que o parto vaginal é um fator de proteção à estas práticas, em contrapartida o parto cesariano é evidenciado como um obstáculo, dados que corroboram com os encontrados na presente pesquisa. Houve também evidência que a presença do Enfermeiro na sala de parto foi um fator intimamente ligado a maior taxa de amamentação precoce, concretizando o quarto passo da IHAC.

Chama atenção a quantidade de crianças, $38,7 \%$, que utilizavam bicos artificiais de maneira precoce, com idade em média de 13,61 dias de vida, prática que traz à criança diversos efeitos negativos no processo da amamentação.

Estes dados corroboram com o estudo de Mendes MLM, et al. (2019), no qual foi possível observar na fala dos pais participantes que as crianças receberam chupeta ainda na maternidade ou dentre os primeiros 15 dias de vida, este item é visto como essencial no enxoval e causa uma imagem positiva socialmente por acalmar o bebê, e isto colabora para a propagação da falsa ideia de benefício no uso dos bicos artificiais.

O uso precoce da chupeta e mamadeira pode favorecer o desmame precoce, pois os mesmos, em virtude de suas utilizações, causam a confusão de bicos nos bebês, prejudicando a pega correta e sucção, fatores fundamentais para o êxito da amamentação. Assim, a realização do aleitamento materno exclusivo é prejudicada, diminuindo a prevalência entre os menores que fazem uso desses bicos artificiais (SANTOS EM, et al., 2019; CARRASCOZA KC, et al., 2014; PELLEGRINELLI ALR, et al., 2015).

A exposição precoce aos bicos artificiais, reflexo do não cumprimento do passo 9 "Orientar as mães sobre o uso e riscos de mamadeiras, bicos e chupetas" da OMS, impacta na saúde infantil pois implica na diminuição do AME, um estudo realizado por De Oliveira Silva OL, et al. (2019), evidenciou que esta prática triplicou as chances da não realização do AME aos 30 dias de vida dos bebês, um fator que justifica este acontecimento é não apenas a exposição aos bicos, e sim a continuidade em seu uso mesmo após a alta hospitalar, intensificando seus efeitos deletérios (WORLD HEALTH ORGANIZATION, 2020).

Os hábitos orais são ações aprendidas que tem direta ligação com o sistema estomatognático e podem tornar-se deletérios dependendo a duração, intensidade e frequência desses atos, dentre os hábitos que podem comprometer este sistema tornando-se deletérios, estão a chupeta e mamadeira.

Pereira TS, et al. (2017), notou em seu estudo que a manutenção dos hábitos orais deletérios por um período de 2 a 4 anos esteve relacionado com alterações da fala e oclusais, consequência da modificação das estruturas musculares envolvidas neste processo.

A pega foi a principal variável que sofreu influência nas crianças que utilizavam bicos artificiais, pois esta estava predominantemente errada nestes casos, seguida da presença de trauma mamilar materno predominantemente nas crianças que utilizavam chupeta e mamadeira. 
Este resultado corrobora com o estudo de Dias JS, et al. (2019), no qual a pega e posicionamento incorretos foram associados ao trauma mamilar na maioria dos estudos analisados em sua revisão integrativa. De acordo com Batista CLC, et al. (2017), o uso de bicos artificiais estava associado a alterações no processo biológico da amamentação, trazendo malefícios para a saúde da criança, principalmente quando relacionado ao desmame precoce, e da mãe quando esta prática traz consigo queixas mamárias e infecções fúngicas, por exemplo.

As consequências da utilização dos bicos artificiais são inúmeras e geralmente são constatadas e estudadas após o primeiro ano de vida, pois é o período em que se tornam mais evidente as alterações e repercussões na saúde infantil. Por este motivo a prática do aleitamento materno deve ser cada vez mais incentivada, pois já está provado seus diversos benefícios à saúde do bebê e também da mãe, no âmbito físico, nutricional e psíquico.

\section{CONCLUSÃO}

Faz-se necessário que haja enfermeiros qualificados para atuar na prevenção dos problemas do uso dos bicos artificiais desencorajando esta prática. Este processo de educação em saúde deve ser exercido, nos diversos cenários que estiver inserido o binômio mãe-bebê, desde o pré-natal até o puerpério, de forma construtivista para esclarecer os efeitos negativos do uso dos bicos e não apenas proibi-la. Com o propósito de reduzir o desmame precoce e repercussões negativas sobre a saúde do bebê.

\section{REFERÊNCIAS}

1. ABDALA LG e DA CUNHA MLC. Contato pele a pele entre mãe e recém-nascido e amamentação na primeira hora de vida. Clinical \& Biomedical Research, 2018; 38(4).

2. BATISTA CLC, et al. Association between pacifier use and bottle-feeding and unfavorable behaviors during breastfeeding. Jornal de Pediatria (Versão em Português), 2018; 94(6): 596-601.

3. BATISTA CLC, et al. Influência do uso de chupetas e mamadeiras na prática do aleitamento materno. Journal of Health \&Biological Sciences, 2017; 5(2): 184-191.

4. Brasil. Ministério da Saúde. Portaria no 569 , de 1ำ de junho de 2000. Institui o Programa de Humanização no Prénatal e Nascimento, no âmbito do Sistema Único de Saúde. Diário Oficial da União, Brasília, DF, 08 jun. 2000. Seção 1, p. 4.

5. Brasil. Ministério da Saúde. Secretaria de Atenção à Saúde. Departamento de Ações Programáticas e Estratégicas. II Pesquisa de Prevalência de Aleitamento Materno nas Capitais Brasileiras e Distrito Federal. Brasília (DF); 2009.

6. Brasil. Ministério da Saúde. Secretaria de Atenção à Saúde. Departamento de Ações Programáticas Estratégicas. Atenção à saúde do recém-nascido: guia para os profissionais de saúde. Brasília (DF); 2014.

7. Brasil. Ministério da Saúde. Secretaria de Atenção à Saúde. Departamento de Atenção Básica. Saúde da criança: aleitamento materno e alimentação complementar (Cadernos de Atenção Básica n. 23). Brasília (DF); 2015.

8. Brasil. Ministério da Saúde. Secretaria de Atenção à Saúde. Estratégia Nacional para Promoção do Aleitamento Materno e Alimentação Complementar Saudável no Sistema Único de Saúde: manual de implementação. Brasília (DF); 2015.

9. BUCCINI GDS, et al. Determinantes do uso de chupeta e mamadeira. Revista de Saúde Pública, 2014; 48(4).

10. CARRASCOZA KC, et al. Fatores determinantes do uso de chupeta entre crianças participantes de programa de incentivo ao aleitamento materno. Rev. CEFAC, 2014; 16(2):582-591.

11. DE ARAÚJO CMT, et al. Aleitamento materno e uso de chupeta: repercussões na alimentação e no desenvolvimento do sistema sensório motor oral. Revista Paulista de Pediatria, 2007; 25(1): 59-65.

12. DE OLIVEIRA SILVA OL, et al. Associação entre oferta de fórmulas infantis e chupetas na maternidade e amamentação nos primeiros seis meses de vida. DEMETRA, 2019; 14: 43555.

13. DIAS EG, et al. Percepção das gestantes quanto à importância das ações educativas promovida pelo enfermeiro no pré-natal em uma unidade básica de saúde. Revista Eletrônica Gestão e Saúde, 2015; (3): 2695-2710.

14. DIAS JS, et al. Fatores associados ao trauma mamilar no período lactacional: uma revisão sistemática. Rev. Bras. Saúde Matern. Infant, 2017;17(1): 43-58.

15. DOMINGUEZ CC, et al. Dificuldades no estabelecimento da amamentação: visão das enfermeiras atuantes nas unidades básicas de saúde. Revista Enfermagem UERJ, 2017; 25: 14448.

16. DOS SANTOS EM, et al. Avaliação do aleitamento materno em crianças até dois anos assistidas na atenção básica do Recife, Pernambuco, Brasil. Ciência \& Saúde Coletiva, 2019; 24(3):1211-1222.

17. MARINHO MS, et al. A atuação do (a) enfermeiro (a) na promoção, incentivo e apoio ao aleitamento materno: revisão bibliográfica. Revista Enfermagem Contemporânea, 2015; 4(2):189-198.

18. MENDES MLM, et al. A influência da reprodução cultural sobre o hábito de sucção de chupeta. Revista Pesquisa Qualitativa, 2019; 7(13): 89-116. 
19. MOREIRA MEL, et al. Práticas de atenção hospitalar ao recém-nascido saudável no Brasil. Cadernos de Saúde Pública, 2014; 30: S128-S139.

20. PELLEGRINELLI ALR, et al. Influência do uso de chupeta e mamadeira no aleitamento materno exclusivo entre mães atendidas em um Banco de Leite Humano. Rev. Nutr, 2015; 28(6):631- 639.

21. PEREIRA TS, et al. Associação entre hábitos orais deletérios e as estruturas e funções do sistema estomatognático: percepção dos responsáveis. CoDAS, 2017; 29(3): e20150301.

22. Portal Ministério da Saúde. Saúde da Criança: o que é, cuidados, políticas, vacinação, aleitamento. Disponível em: http://portalms.saude.gov.br/saude-de-a-z/crianca. Acesso em 18 fev 2019.

23. REA MF. Os benefícios da amamentação para a saúde da mulher. J Pediatr (Rio J), 2004; 80(5): S142-6.

24. SILVA JLP, et al. Fatores associados ao aleitamento materno na primeira hora de vida em um hospital amigo da criança. Texto Contexto Enferm, 2018; 27(4): e4190017.

25. Sociedade Brasileira de Pediatria. CIENTÍFICO C, ELIAS CLLF. Uso de chupeta em crianças amamentadas: prós e contras. Guia prático de atualização. 2017

26. TOMA TS, REA MF. Benefícios da amamentação para a saúde da mulher e da criança: um ensaio sobre as evidências. Cadernos de Saúde Pública, Rio de Janeiro, 2008; 24: 235-246.

27. World Health Organization. 2020. Ten steps to successful breastfeeding. Disponível em: http://www.who.int/nutrition/bfhi/ten-steps/en/. Acesso em 10 out. 2020. 